
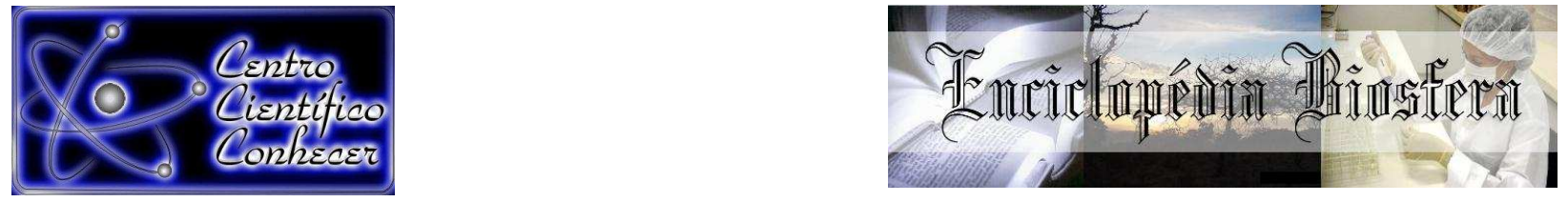

\title{
EFEITO DO SOMBREAMENTO NA GERMINAÇÃO E DESENVOLVIMENTO DE MUDAS DE CANZILEIRO (Platypodium elegans Vog)
}

Sue Éllen Ester Queiroz ${ }^{1}$, Gérsyca Eliana Aparecida Gonçalves Mendes², Ademir Martins Pereira Júnior², Paulo Henrique Sales Guimarães ${ }^{3}$

1. Departamento de Biologia - Instituto Federal do Sul de Minas - Câmpus Machado. Rodovia Machado - Paraguaçú, km 3 - Bairro Santo Antônio Machado - MG - CEP 37.750-000 - Email: sue.queiroz@ifsuldeminas.edu.br, sueellenqueiroz@yahoo.com.br

2. Tecnólogo em Gestão Ambiental pelo Instituto Federal Goiano - Câmpus Urutaí

3. Professor do Departamento de Estatística - Universidade Federal de Lavras

Recebido em: 08/09/2015 - Aprovado em: 14/11/2015 - Publicado em: 01/12/2015 DOI: http://dx.doi.org/10.18677/Enciclopedia_Biosfera_2015_144

\section{RESUMO}

A produção de mudas de espécies nativas tem se tornado cada vez mais importante para a recomposição de florestas degradadas, porém a falta de trabalhos relacionados para muitas espécies dificulta a obtenção de mudas de qualidade, como é o caso de Platypodium elegans Vog. Sendo assim, o presente trabalho teve como objetivo avaliar o efeito do sombreamento na produção de mudas de $P$. elegans. A pesquisa foi conduzida no viveiro do Instituto Federal Goiano - Câmpus Urutaí, GO, foram avaliados quatro níveis de sombreamento, 0 (pleno sol), 30, 50 e $70 \%$ no processo de germinação, sobrevivência e desenvolvimento das mudas. $O$ delineamento utilizado foi o de blocos casualizados com quatro repetições, contendo 30 plantas por parcela. A contagem de germinação foi realizada semanalmente e a sobrevivência das mudas foi quantificada ao final do experimento. Após 120 dias de permanência no viveiro as mudas foram avaliadas quanto à altura, diâmetro do coleto, número de folhas, número de folíolos, comprimento da raiz principal, número de raízes secundárias, peso seco da parte aérea e peso seco da raiz. Os resultados demonstraram que o sombreamento interferiu no desenvolvimento das mudas, havendo assim melhores resultados naquelas que se encontravam a pleno sol, com aumento da germinação, sobrevivência, índice de velocidade de emergência (IVE), peso seco e comprimento da raiz. Ao término do experimento foi possível concluir que a produção de mudas de $P$. elegans deve ser realizada a pleno sol, desde a semeadura até a fase final de desenvolvimento das mudas.

PALAVRAS-CHAVE: Cerrado, espécie pioneira, produção de mudas.

\section{EFFECT OF SHADE ON SEEDS GERMINATION AND CANZILEIRO SEEDLINGS DEVELOPMENT (Platypodium elegans Vog)}

\begin{abstract}
The production of seedlings of native species has become increasingly important for the restoration of degraded forests, but the lack of related work for many species difficult to obtain quality seedlings, as is the case of Platypodium elegans Vog.
\end{abstract}


Therefore, this study aimed to evaluate the effect of shading in the production of $P$. elegans seedlings. The research was conducted in the nursery of the Federal Institute Goiano - Campus Urutaí, GO, were evaluatede four shading levels 0 (full sun), 30, 50 and $70 \%$ in the process of germination, survival and development of the seedlings. The design was a randomized block with four replications, with 30 plants per plot. Germination counts were obtained weekly and survival of seedlings was quantified at the end of the experiment. After 120 days of stay in the nursery seedlings were evaluated for height, stem diameter, number of leaves, number of leaflets, main root length, number of secondary roots, dry weight of shoot and root dry weight. The results showed that the shading interfered with the development of the plants, thus having better results in those who were in full sun, with increased germination, survival, emergency speed index (IVE), dry weight and root length. At the end of experiment it was concluded that the production of $P$. elegans seedlings in full sun should be performed, from seeding until the final stage of development of the seedlings.

KEYWORDS: Cerrado, pioneer species, seedling production.

\section{INTRODUÇÃO}

O Cerrado ocupa uma área de 204,7 milhões de hectares na porção central do Brasil e engloba parte dos estados da Bahia, Goiás, Maranhão, Mato Grosso, Mato Grosso do Sul, Minas Gerais, Paraná, Piauí, São Paulo e Tocantins, além do Distrito Federal (IBGE, 2004). Com o aumento das áreas de produção agrícola e a inclusão de novas tecnologias para a exploração das terras para a agricultura, grande parte da biodiversidade do cerrado tem diminuído, levando a extinção de várias espécies tanto da flora quanto da fauna (KLINK \& MACHADO, 2005).

Platypodium elegans Vog, popularmente conhecido como canzileiro, amendoim-do-campo, faveiro e uruvalheira é uma espécie nativa do cerrado pertencente a família Leguminosae-Papilionoideae. E uma planta pioneira, semidecídua, heliófita, com características do cerrado localizado em terrenos bem drenados e em sua transição para a floresta semidecídua (LORENZE, 2000). Mas apesar da importância da espécie para recuperação de áreas degradadas e paisagismo, encontram-se poucos trabalhos relacionados à propagação da espécie, dificultando a produção de mudas de qualidade.

Segundo DELARMELINA et al. (2014), estudos relacionados a produção de mudas com o intuito de recuperar áreas impactadas possui extrema importância para a preservação ambiental. Sendo que, a caracterização das condições mais apropriadas para a produção de mudas propicia melhor crescimento inicial em campo, colaborando para o aumento da homogeneidade, sanidade e redução da mortalidade do plantio (FERREIRA et al., 2009).

Em muitos viveiros a produção de mudas de espécies florestais tem sido realizada em pleno sol, visando melhorar a adaptação das plantas às condições de campo e economizar na estrutura. No entanto alguns viveiristas, ainda mantém a tradição de formação de mudas com $50 \%$ de sombreamento, sendo tais recomendações utilizadas há muito tempo e se tornaram padrão nos viveiros florestais (PAIVA et al., 2003). Porém, sabe-se que, cada espécie comporta de maneira diferente no que se refere à luminosidade (DAVID \& SILVA, 2008).

Várias classificações, em relação à adaptação das espécies a luminosidade, têm sido propostas na literatura especializada, sendo que o mais empregado é a classificação em quatro grupos distintos: pioneiras, secundária inicial, secundária tardia e clímax. Os grupos de espécies adaptadas a condições de maior 
luminosidade colonizam as áreas abertas e crescem rapidamente, fornecendo o sombreamento necessário para o crescimento de espécies mais tardias, sendo que a tolerância das espécies ao sombreamento aumenta das pioneiras para as clímax (MARTINS, 2007). Assim, espécies pioneiras se desenvolvem melhor em condições de plena luminosidade.

O sombreamento artificial em viveiros, feito por meio de telas de polietileno que proporcionam diferentes níveis de passagem de luz, já foi apontado em vários estudos como uma técnica que auxilia o crescimento das mudas. SANTOS et al. (2014) observaram que o sombreamento de 30 e $50 \%$ levou a incrementos significativos na altura das mudas de Ochroma pyramidale Cav. Também SANTOS et al. (2010), recomendam o sombreamento de 30\% para a produção de mudas de Eucalyptus grandis Hill, o que além proporcionar ganhos em altura, também favorece o aumento da matéria seca das folhas, coleto e raiz.

Estudos realizados em Eugenia uniflora Linn. evidenciaram que o crescimento inicial das mudas foi favorecido pelas condições de pleno sol (MARTINAZZO et al., 2007). MOTA et al. (2013) também encontram resultados semelhantes para a produção de mudas de Anadenanthera falcata Benth. Sspeg. No entanto, pesquisas relacionadas para $P$. elegans ainda não foram descritas na literatura. Sabendo-se que o sombreamento é um fator importante no processo de produção de mudas, o presente trabalho teve como objetivo avaliar o efeito do sombreamento na germinação das sementes e desenvolvimentos inicial das plantas de $P$. elegans.

\section{MATERIAL E MÉTODOS}

O presente trabalho foi realizado no Viveiro Florestal do Instituto Federal Goiano - Câmpus Urutaí. Os frutos foram coletados no município de Urutaí, na

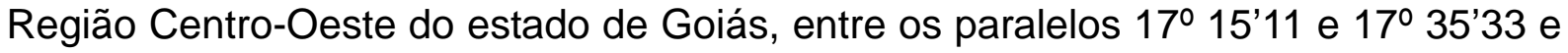

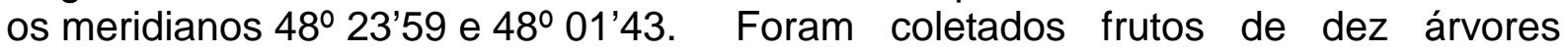
matrizes, no ano de 2011, nos meses de setembro e outubro, sendo a coleta dos frutos realizada manualmente, coletando apenas os frutos maduros, antes da dispersão da planta-mãe. Após a coleta os frutos foram escarificadas com auxilio de um esmeril, em seguida foram semeadas em sacos plásticos de capacidade de 2 $\mathrm{dm}^{3}$, contendo substrato preparado a partir de $50 \%$ de terra de subsolo, $25 \%$ de areia, $25 \%$ de húmus de minhoca e $400 \mathrm{mg} \mathrm{dm}^{-3}$ de superfosfato simples (Tabela 1 ).

TABELA 1. Características químicas do substrato utilizado na produção de mudas de $P$. elegans.

\begin{tabular}{|c|c|c|c|c|c|c|}
\hline $\mathrm{pH} \mathrm{H} \mathrm{H}_{2} \mathrm{O}$ & $\mathrm{P}$ & $\mathrm{K}$ & $\mathrm{Al}^{3+}$ & $\mathrm{H}^{+} \mathrm{AL}$ & $\mathrm{Ca}^{2+}+\mathrm{Mg}^{2+}$ & CTC \\
\hline & \multicolumn{2}{|c|}{$\mathrm{mg} \mathrm{dm}^{-3}$} & \multicolumn{4}{|c|}{$\mathrm{Cmol}_{\mathrm{C}} \mathrm{Kg}^{-1}$} \\
\hline 7,22 & 480 & 713 & 0,0 & 0,7 & 7,3 & 9,8 \\
\hline
\end{tabular}

Em cada recipiente foram colocadas três sementes recobertas com uma fina camada do mesmo substrato. Em seguida os recipientes foram submetidos a quatro níveis de sombreamento, aplicados por meio de telas de polietileno de $0 \%$ (pleno sol), $30 \%, 50 \%$ e $70 \%$, em telados com pé-direito de $50 \mathrm{~cm}$ de altura, com recobrimento lateral. $O$ delineamento experimental utilizado foi 0 de blocos casualizados com quatro repetições de 30 mudas. Trinta dias após a semeadura foi realizado um desbaste, com o objetivo de eliminar as plantas jovens excedentes em cada recipiente, deixando-se apenas a mais central e vigorosa.

As avaliações de emergência foram realizadas semanalmente a partir do $7^{\circ}$ 
dia da semeadura e prosseguiram até a taxa de germinação mostrar-se constante. Foi avaliada a porcentagem final da germinação e o Índice de Velocidade de Emergência (IVE), empregando a fórmula de MAGUIRE (1962) utilizando-se a Equação 1.

$\operatorname{IVE}=(E 1 / \mathrm{N} 1)+(\mathrm{E} 2 / \mathrm{N} 2)+\ldots+(\mathrm{En} / \mathrm{Nn})$

em que contagem.

$E 1, E 2, E n=$ número de plântulas emergidas na primeira, segunda e última

$\mathrm{N} 1, \mathrm{~N} 2, \mathrm{Nn}=$ dias de embebição na primeira, segunda e última contagem.

Após 120 dias da semeadura as mudas foram levadas ao Laboratório de Sementes do Instituto Federal Goiano - Câmpus Urutaí no qual foi mensurada a altura das mudas, parte aérea e comprimento da raiz principal, com o auxílio de uma régua graduada em milímetros, para determinar o diâmetro do coleto foi utilizado um paquímetro digital. $\mathrm{O}$ número de folhas, folíolos e raiz secundária também foram quantificados.

As plantas foram desagregadas do substrato, com uso de água corrente, para a avaliação das raízes das mudas, sendo posteriormente exposta sobre a bancada do laboratório a temperatura ambiente para a retirada do excesso de água. Logo em seguida utilizou-se estufa regulada à $9^{\circ} \mathrm{C}$ por três dias para determinar o teor de massa seca referente a parte aérea e raiz. Os resultados obtidos foram submetidos à análise de variância, sendo que as médias foram comparadas entre si pelo teste de Tukey a $5 \%$ de probabilidade utilizando o programa SISVAR 5.3 ( 2011).

\section{RESULTADOS E DISCUSSÃO}

Ao analisar a Tabela 2 é possível observar que após as avaliações de germinação realizadas em campo notou-se que as sementes de $P$. elegans germinam em qualquer situação, tanto a pleno sol quanto em diferentes níveis de sombreamento testados. Entretanto os resultados de germinação a pleno sol foram superiores aos demais tratamentos. Os melhores índices de velocidade de emergência também foram obtidos nas mudas crescidas a pleno sol. Apesar de haver germinação com sombreamento, foram encontrados problemas nesta condição em relação à sobrevivência das mudas, sendo que obteve-se $96,2 \%$ de sobrevivência a pleno sol e apenas $57,5 \%$ no sombreamento a $70 \%$. Os resultados de sobrevivência no sombreamento de $70 \%$ foram inferiores aos demais tratamentos $(0 \%, 30 \%$ e $50 \%)$, o quê sugere intolerância das plantas ao sombreamento. Estes resultados corroboram com os de LORENZE (2000), que afirma que as sementes de $P$. elegans germinam melhor a pleno sol.

TABELA 2. Valores médios de germinação em porcentagem, índice de velocidade de emergência (IVE) e sobrevivência após 120 dias de semeadura de Platypodium elegans em diferentes níveis de sombreamento.

\begin{tabular}{cccc}
\hline Níveis de Sombreamento & Germinação (\%) & IVE & Sobrevivência (\%) \\
\hline $0 \%$ & $23,50( \pm 1,2) \mathrm{a}$ & $27,98( \pm 11,3) \mathrm{a}$ & $96,25( \pm 0,9) \mathrm{a}$ \\
$30 \%$ & $11,25( \pm 0,8) \mathrm{ab}$ & $11,85( \pm 10,3) \mathrm{b}$ & $61,28( \pm 0,9) \mathrm{b}$ \\
$50 \%$ & $10,06( \pm 0,5) \mathrm{ab}$ & $13,20( \pm 9,8) \mathrm{ab}$ & $97,72( \pm 0,4) \mathrm{a}$ \\
$70 \%$ & $4,75( \pm 0,6) \mathrm{b}$ & $5,49( \pm 4,2) \mathrm{b}$ & $57,50( \pm 0,3) \mathrm{b}$ \\
\hline
\end{tabular}

Médias seguidas pela mesma letra na linha não diferem entre si pelo Teste de Tukey, ao nível de $5 \%$ de probabilidade. 
A análise de variância evidenciou, na Tabela 3, que houve diferença entre as médias dos tratamentos nos parâmetros de comprimento de raiz e peso seco da raiz. As mudas de $P$. elegans apresentaram valores semelhantes nos parâmetros de diâmetro, altura total, número de folhas, folíolos, peso seco da parte aérea, e raiz secundária nos diferentes níveis de sombreamento avaliados. Porém, o maior comprimento da raiz principal foi obtido nas condições de pleno sol quando comparadas às plantas que cresceram com distintos sombreamentos.

TABELA 3. Valores médios do diâmetro (Ǿ) do coleto, comprimento da raiz principal, altura da muda, número de folhas, número de folíolos, peso seco (PS) da parte aérea e, peso seco (PS) da raiz das mudas de Platypodium elegans formadas a partir de diferentes níveis de sombreamento.

\begin{tabular}{cccccc}
\hline & \multicolumn{5}{c}{ Níveis de Sombreamento } \\
\cline { 2 - 6 } Avaliações & $\mathbf{0 \%}$ & $\mathbf{3 0 \%}$ & $\mathbf{5 0 \%}$ & $\mathbf{7 0 \%}$ & $\mathbf{C V}(\%)$ \\
\hline Ǿ Coleto $(\mathrm{mm})$ & $2,77( \pm 0,64) \mathrm{a}$ & $3,07( \pm 0,22) \mathrm{a}$ & $2,90( \pm 0,44) \mathrm{a}$ & $2,33( \pm 0,34) \mathrm{a}$ & 13,89 \\
Comp. Raiz $(\mathrm{cm})$ & $9,80( \pm 3,8) \mathrm{a}$ & $5,37( \pm 1,3) \mathrm{ab}$ & $4,97( \pm 2,1) \mathrm{b}$ & $7,33( \pm 4,3) \mathrm{a}$ & 23,23 \\
Raiz Secundária & $7,70( \pm 7,1) \mathrm{a}$ & $4,97( \pm 2,5) \mathrm{a}$ & $6,77( \pm 4,3) \mathrm{a}$ & $5,83( \pm 3,6) \mathrm{a}$ & 55,55 \\
Altura $(\mathrm{cm})$ & $19,52( \pm 5,6) \mathrm{a}$ & $16,22( \pm 1,6) \mathrm{a}$ & $15,80( \pm 3,5) \mathrm{a}$ & $18,15( \pm 6,7) \mathrm{a}$ & 12,82 \\
№ Folhas & $3,92( \pm 1,8) \mathrm{a}$ & $3,35( \pm 0,4) \mathrm{a}$ & $2,52( \pm 1,1) \mathrm{a}$ & $3,16( \pm 1,6) \mathrm{a}$ & 25,95 \\
№ Folíolos & $21,47( \pm 10,7) \mathrm{a}$ & $18,02( \pm 2,6) \mathrm{a}$ & $13,47( \pm 7,00) \mathrm{a}$ & $14,73( \pm 6,9) \mathrm{a}$ & 27,95 \\
PS Aérea $(\mathrm{g})$ & $0,19( \pm 0,09) \mathrm{a}$ & $0,19( \pm 0,02) \mathrm{a}$ & $0,15( \pm 0,09) \mathrm{a}$ & $0,14( \pm 0,04) \mathrm{a}$ & 24,5 \\
PS Raiz $(\mathrm{g})$ & $0,18( \pm 0,1) \mathrm{a}$ & $0,15( \pm 0,02) \mathrm{ab}$ & $0,15( \pm 0,07) \mathrm{ab}$ & $0,08( \pm 0,03) \mathrm{b}$ & 27,72 \\
\hline
\end{tabular}

Médias seguidas pela mesma letra na linha não diferem entre si pelo Teste de Tukey, ao nível de $5 \%$ de probabilidade.

MUROYA et al. (1997), estudando a análise de crescimento das mudas de jacareúba (Calophyllum angulare) cultivadas em condição de viveiro observou que a altura das mudas possui diferentes padrões de respostas de acordo com a capacidade adaptativa da espécie a variações na intensidade de luz. AGUIAR et al. (2005) também não observaram diferenças significativas nos valores de altura do pau-brasil (Caesalpinia echinata) nos diferentes níveis de sombreamento estudado em relação a pleno sol, igualmente aos resultados obtidos neste trabalho para $P$. elegans. Segundo ZANELLA et al. (2006) a adaptação das plantas ao ambiente de luz depende de seu sistema fotossintético, de modo que a luminosidade ambiental seja empregada de maneira mais eficiente possível, sendo, as respostas dessa adaptação refletidas no desenvolvimento total da planta.

O maior comprimento da raiz foi obtido a pleno sol, o que pode ser explicado por MOTA et al. (2013), pelo fato de que as plantas perdem mais água por evapotranspiração a pleno sol, o que leva a redução da disponibilidade hídrica para as raízes, forçando a planta a translocar mais assimilados para o sistema radicular, resultando em maior crescimento das raízes para a absorção de água nas camadas mais profundas do solo. O peso seco da raiz foi superior nas condições de pleno sol com $0,18 \mathrm{~g}$, quando comparado aos demais tratamentos. Isto se deve ao comprimento da raiz principal, que também foi superior na condição de pleno sol e em sombreamento $70 \%$.

Segundo SILVA et al. (2007), plantas expostas a uma maior intensidade de iluminação apresentam maior acúmulo de massa seca na raiz, permitindo maior ENCICLOPÉDIA BIOSFERA, Centro Científico Conhecer - Goiânia, v. 11 n.22; p. 1080 
absorção de água e nutrientes, estratégia que garante que a planta tenha capacidade de suportar taxas elevadas de fotossíntese e transpiração em ambientes mais iluminados. CARVALHO FILHO et al. (2002) estudaram o desenvolvimento de mudas de canafístula (Cassia grandis L.) e observaram que as plantas submetidas ao sombreamento apresentaram maior número de folhas, altura, diâmetro de caule e peso de matéria seca de folha, caule e raiz em relação às que permaneceram a pleno sol.

As diferentes condições de luminosidade ocasionam, em geral, variações morfológicas e fisiológicas na planta, sendo que o grau de adaptação é ditado por suas características genéticas em interação com seu ambiente e, as consequências dessas diferenças de intensidade de luz são mais significativas no crescimento da planta do que na sua qualidade, principalmente no que diz respeito ao acúmulo de matéria seca em condições naturais (SCALON et al., 2003). Segundo ALMEIDA (2005) o sombreamento induz as espécies vegetais a desenvolver táticas de ganho de área foliar para maior absorção dos raios luminosos.

\section{CONCLUSÕES}

As condições a pleno sol proporcionaram aumento da germinação, sobrevivência, índice de velocidade de emergência (IVE), peso seco e comprimento da raiz das mudas de $P$. elegans. Podendo-se concluir que as mudas de $P$. elegans se desenvolvem melhor nas condições de pleno sol, dispensando o sombreamento desde a semeadura até a produção final da muda.

\section{REFERÊNCIAS}

ALMEIDA, L. S. Avaliação morfológica de mudas de Allophylus edulis (a. st.-hil., a. juss. \& cambess.) radl. (vacum) e Schinus terebinthifolius raddi (aroeira) produzidas em diferentes substratos. 2005. 105f. Dissertação (Mestrado em Ciências Florestais) - Universidade Federal do Paraná, Curitiba, 2005.

AGUIAR, F. F. A.; KANASHIRO, S.; TAVARES, A. R.; PINTO, M. M.; STANCATO, G. C.; AGUIAR, J.; NASCIMENTO, T. D. R. Germinação de sementes e formação de mudas de Caesalpinia echinata Lam. (pau-brasil): efeito de sombreamento. Revista Árvore, Viçosa-MG, v. 29, n. 6, p. 871-875, 2005.

CARVALHO FILHO, J. L. S.; BLANK, M. F. A.; BLANK, A. F.; SANTOS NETO, A. L.; AMÂNCIO, V. F. Produção de mudas de Cassia grandis L. em diferentes ambientes, recipientes e misturas de substratos. Revista Ceres, v. 49, n. 284, p. 341-352, 2002.

DAVID, A. C.; SILVA, E. A. A. Produção de sementes e mudas de espécies florestais. Lavras: UFLA, 2008, $175 \mathrm{p}$.

DELARMELINA, W. M.; CALDEIRA, M. V. W.; FARIA, J. C. T.; GONÇALVES, E. O.; ROCHA, R. L. F. Diferentes substratos para a produção de mudas de Sesbania virgata. Floresta Ambiente, v. 21, n. 2, p. 224-233, 2014.

FERREIRA, D. F. Sisvar: a computer statistical analysis system. Ciência e Agrotecnologia (UFLA), v. 35, n. 6, p. 1039-1042, 2011.

FERREIRA, M. G. R; ROCHA, R. B.; GONÇALVES, E. P.; ALVES, E. U.; RIBEIRO, G. D. Influência do substrato no crescimento de mudas de cupuaçu (Theobroma 
grandiflorum Schum.) Acta Sciencentiarum Agronomy, v. 31, n. 4, p. 677-681, 2009.

IBGE. Mapa de biomas do Brasil. Escala 1:5.000.000. Rio de Janeiro: IBGE, 2004. Disponível em: http://mapas.ibge.gov.br/biomas2/viewer.htm. Acesso em: $19 \mathrm{fev}$. 2015.

KLINK, C. A.; MACHADO, R. B. A conservação do cerrado Brasileiro. Megadiversidade, v. 1, n. 1, p. 147-155, 2005.

LORENZE, H. Árvores brasileiras: manual de identificação e cultivo de plantas arbóreas nativas do Brasil. 3ª̣ed. Vol. 1. Nova Odessa, Brazil, 2000.

MAGUIRE, J.D. Speed of germination-aid in selection and evaluation for seedling emergence and vigor. Crop Science, v. 1, p. 176-177, 1962.

MARTINAZZO, E. G.; ANESE, S.; WANDSCHEER, A. C. D.; PASTORINI, L. H. Efeito do sombreamento sobre o crescimento inicial e teor de clorofila foliar de Eugenia uniflora Linn (Pitanga) - Família Myrtaceae. Revista Brasileira de Biociências, Porto Alegre, v. 5, supl. 2, p. 162-164, jul. 2007.

MARTINS, S. V. Recuperação de matas ciliares. Viçosa, 2007.

MOTA, L. H. S.; SCALON, S. P.Q.; MUSSURY, R. M. Efeito do condicionamento osmótico e sombreamento na germinação e no crescimento inicial das mudas de angico (Anadenanthera falcata Benth. Speg.). Revista Brasileira de plantas medicinais, v. 14, n. 4, p.655-653, 2013.

MUROYA, K.; VARELA, V. P.; CAMPOS, M. A. A. Análise de crescimento das mudas de jacareúba (Calophyllum angulare A. C. Smith - Guttiferae) cultivadas em condição de viveiro. Acta Amazônica, v. 27, n. 3, p. 197-212, 1997.

PAIVA, L. C.; GUIMARÃES, R. J.; SOUZA, C. A. S. Influencia de diferentes níveis de sombreamento sobre 0 crescimento de mudas de cafeeiro. Ciência e agrotecnologia, v. 27, n. 1, p. 134-140, 2003.

SANTOS, R. F.; MORAIS, L.; BORSOI, A.; SECCO, D.; MOREIRA, G. C. Shading levels on production and development of Eucalyptus grandis Hill ex Maiden seedlings. Pesquisa Aplicada \& Agrotecnologia, v. 3, n. 3, p.207-2013, 2010.

SANTOS, U. F.; XIMENES, F. S.; LUZ, P. B.; SEABRA JUNIOR, S.; PAIVA SOBRINHO, S. Níveis de sombreamento na produção de mudas de pau-de-balsa (Ochroma pyramidale). Bioscience Journal, v. 30, n. 30, p. 129-136, 2014.

SCALON, S. P. Q.; MUSSURY, R. M.; RIGONI, M. R.; SCALON FILHO, H. Crescimento inicial de mudas de Bombacopsis glabra (Pasq.) A. Robyns sob condições de sombreamento. Revista Árvore, v. 27, n. 06, p. 753-758, 2003.

SILVA, B.M.S.; LIMA, J.D.; DANTA, V.A.V.; MORAES, W.S.; SABONARO, D.Z. Efeito da luz no crescimento de mudas de Hymenaea parvifolia Huber. Revista Árvore, v. 
31, n. 6, p. 1019-1026, 2007.

ZANELLA, F.; SONCELA. R.; LIMA. A. L. S. Formação de mudas de maracujazeiro "amarelo" sob níveis de sombreamento em Ji-Paraná/RO. Ciência e agrotecnologia, v. 30, n. 5, p. 880-884, 2006. 